Methods: Consecutive patients with knee OA undergoing primary TKA, extracted from the Longitudinal Leiden Orthopaedics and Outcomes of OsteoArthritis Study (LOAS Study), were included. Self-reported knee joint instability and the Knee injury and Osteoarthritis Outcome Score (KOOS) Pain, Activity Daily Living (ADL) and QoL subscales ( $0-100$; worst-best) were assessed by questionnaires prior and one year after surgery. Multivariable regression analyses were performed to determine associations between knee joint instability, pain, activity limitations and quality of life, adjusted for potential confounders including age, sex, comorbidities, physical activity and preoperative frailty.

Results: 908 patients were included of which 649 patients $(72 \%)$ reported preoperative knee joint instability (mean age 67 years (SD8.6), 453 females $(70 \%))$ and 187 patients $(21 \%)$ postoperative knee joint instability. Preoperative knee joint instability was associated with preoperative KOOS Pain (B-7.2;95\% Cl10.9-3.5) and ADL (B3.8;95\% Cl-7.5-0.09), but not QoL (B-0.4;95\% Cl-2.1-1.2). In addition, postoperative knee joint instability was associated with postoperative KOOS Pain (B-13.5;95\% Cl-17.0-10.0), ADL (B-15.1,95\% Cl-18.4-11.8) and QoL (B-11.0;95\% Cl-13.5-8.5). Among the patients with preoperative self-reported knee joint instability, 165 patients $(25 \%)$ retained knee instability and among the patients with no preoperative self-reported knee joint instability, $22(8 \%)$ developed knee instability one year after surgery. After adjusting for baseline scores and potential confounders, retained knee joint instability was associated with postoperative KOOS Pain (B-19.6;95\% Cl-30.9-8.3), ADL (B-16.5;95\% Cl-27.0-5.9) and QoL (B-13.0;95\% Cl-17.9-8.1).

Conclusions: In usual care, knee joint instability is prevalent one year after TKA $(21 \%)$. Reported knee joint instability is associated with more pain, worse physical function (pre- and postoperatively) and worse Qol postoperatively. Besides, retained knee joint instability was associated with worse pain, physical function and QoL. This emphasizes the importance of further research into the genesis of pre- and postoperative knee joint instability.

Acknowledgements: The study was funded by the Dutch Arthritis Foundation (DAF).

Disclosure of Interest: None declared

DOI: 10.1136/annrheumdis-2017-eular.5988

\section{SAT0506 EARLY POSTOPERATIVE IDENTIFICATION OF PATIENTS AT RISK FOR UNFAVOURABLE LONG-TERM OUTCOMES}

C. Leichtenberg ${ }^{1}$, H.H. Kaptijn ${ }^{2}$, S.H. Verdegaal ${ }^{3}$, R. Onstenk ${ }^{4}$, H.M. van der Linden-van der Zwaag ${ }^{1}$, S.B. Vehmeijer ${ }^{5}$, W.-J.C. Marijnissen ${ }^{6}$, P.-J. Damen ${ }^{7}$, R.G. Nelissen ${ }^{1}$, T.P. Vliet Vlieland ${ }^{1}$, M.G. Gademan ${ }^{1}$ on behalf of LOAS Studygroup. ${ }^{1}$ Orthopedics, Leiden University Medical Center, Leiden; 2 Orthopedics, LangeLand Hospital, Zoetermeer; ${ }^{3}$ Orthopedics, Alrijne Hospital, Leiden and Leiderdorp; ${ }^{4}$ Orthopedics, Groene Hart Hospital, Gouda;

${ }^{5}$ Orthopedics, Reinier de Graaf Hospital, Delft; ${ }^{6}$ Orthopedics, Albert Schweitzer Hospital, Dordrecht; ${ }^{7}$ Orthopedics, Waterlandziekenhuis, Purmerend, Netherlands

Background: Identification of patients who benefit from Total Hip or Knee Arthroplasty (THA or TKA) is necessary to indicate the need for surgery. This seems difficult as $20 \%$ of all patients report unfavourable outcomes. To provide better treatment options for patients with unfavourable outcomes, they should be identified at an early stage after surgery.

Objectives: I To investigate the prevalence of four trajectories in THA and TKA patients for both pain and function separately: (1) Patients with favourable initial and long-term outcomes, (2) patients with favourable initial but unfavourable long-term outcomes, (3) patients with unfavourable initial and favourable long-term outcomes and (4) patients with unfavourable initial and long-term outcomes. II To determine if long-term outcomes in all patients and in patient groups stratified by initial outcome (favourable/unfavourable) can be predicted on the basis of preand early postoperative variables.

Methods: Prospective, multicentre, observational study including consecutive patients undergoing primary THA and TKA (Longitudinal Leiden Orthopaedics and Outcomes of OsteoArthritis Study (LOAS). Measurements were done preoperatively and at 6 and 12 months. Pain and function were assessed with the Hip Disability/ Knee injury and Osteoarthritis Outcome Scores (HOOS or KOOS) Pain and Activity Daily Living (ADL) subscales (score range 0-100). In addition, preoperative variables (sex, age, comorbidities) and early postoperative complications (reoperations or readmissions) were gathered by questionnaire. Scores of the Pain and ADL subscales above and below 70 were defined as favourable and unfavourable outcomes, whereas initial and long-term outcomes were defined as 6 months and 12 months after surgery, respectively. First the prevalence's of the trajectories were determined. Secondly, multivariable logistic regression analysis were done to examine if preoperative variables, postoperative complications and initial outcomes predicted long-term pain and function outcomes. Identical analyses were performed in the patients with favourable and unfavourable initial outcomes separately.

Results: 972 THA and 892 TKA patients were included. With respect to pain, the prevalence of trajectories $1,2,3$ and 4 were $79 \%(n=740), 5 \%(n=47), 7 \%$ $(n=63)$ and $9 \%(n=88)$ in THA and $75 \%(n=606), 4 \%(n=33), 9 \%(n=71)$ and $12 \%$ $(n=93)$ for TKA. With respect to function, the prevalence's were $74 \%(n=696), 6$ $(n=53), 7 \%(n=63)$ and $14 \%(n=131)$ for THA and $72 \%(n=624), 5 \%(n=49), 9 \%$ $(n=78)$ and $14 \%(n=119)$ for TKA, respectively. Moreover, one-third of the patients with initial unfavourable pain or function outcomes attained favourable long-term outcomes. Overall, only the initial postoperative HOOS/KOOS pain and function predicted long-term outcomes (Table 1).

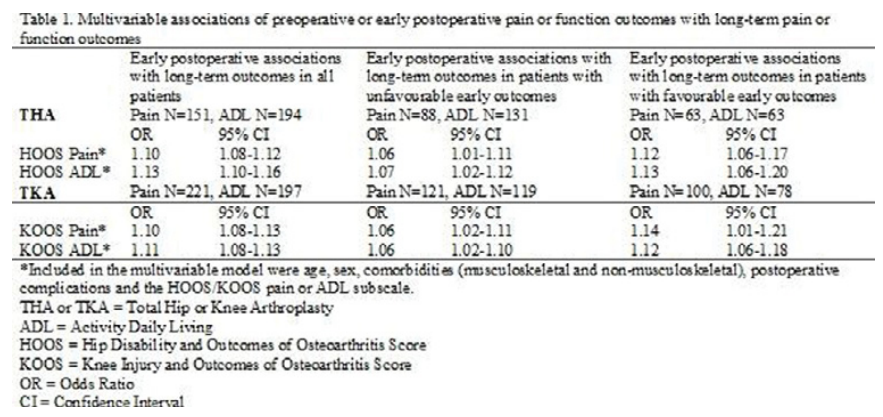

Conclusions: One-third of patients with unfavourable initial outcomes improved further after the first six months after surgery. More research into the recovery early after surgery may help to identify patients at risk for unfavourable outcomes and modify their course by appropriate interventions.

Acknowledgements: This study was funded by the Dutch Arthritis Foundation. Disclosure of Interest: None declared

DOI: 10.1136/annrheumdis-2017-eular.2295

\section{SAT0507 OXYTOCINE IN OSTEOARTHRITIS: FROM IN VITRO TO IN VIVO}

C.H. Roux ${ }^{1}$, E. Fontas ${ }^{2}$, L. Euller-Ziegler ${ }^{3}$, V. Breuil ${ }^{3}$, E.Z. Amri ${ }^{4}$. ${ }^{1}$ Rheumatology, University of Nice Sofia Antipolis, IBV; ${ }^{2}$ Biostatistical Department; ${ }^{3}$ Rheumatology, CHU Nice, University Nice Sofia Antipolis; ${ }^{4}$ Biological Department, University of Nice Sofia Antipolis, IBV, nice, France

Background: The relationship between oxytocine (OT) and osteoarthritis (OA) is poorly studied and remains unknown. However the subchondral bone is considered as a major player in osteoarthritis, and a protective effect of OT on bone mineralization is described in recent studies.

Objectives: The aim of our work was to investigate a possible link between OT and $\mathrm{OA}$

Methods: we used three different approaches: i) In vitro using MSC (adipose and bone marrow derived stem cells) that were maintained as mono-layer (2D) or as pellets in three dimension (3D) in the absence or the presence of OT. RT-PCR, histological and immunohistochemical analysis were performed. ii) in vivo using a rat animal model developing $O A$ upon anterior cruciate ligament transection (ACLT). Twenty female Wistar rats were divided in 2 groups: one group was sham operated whereas the second was ACL transected. Half of each group received either OT ( $1 \mathrm{mg} / \mathrm{kg} /$ day) injections or saline buffer. Histological analysis was performed on day 28 . The severity of OA lesions was assessed on a scale adapted from Mankin's score at medial part of the femur. iii) Clinical analysis using a human cohort. Samples issued from a hand OA cohort (ADEM). 63 OA women have been included; men were excluded because of their low number. At baseline, all subjects benefited of OT and leptin circulating levels measurements and hand $X$ rays (Scoring systems including Kelgren and Lawrence and Verbruggen scores). The control group consists in subjects of the same age range without osteoporosis, OA or inflammatory disease.

Results: OT treatment of differentiating MSCs for 21 days induced a significant increase of agrecan, Col X and COMP mRNA levels without variation of Col 1a. Immunostaining experiments showed an improvement of Sox9 and Col II expression in the presence of OT. The histological analysis of rat knee showed no differences in the severity of lesions between the different ACLT groups. The characteristics of the women cohorts is as follow: 63 OA women age $=65(+/-11)$, Body Mass Index $(\mathrm{BMI})=24(+/-4), \mathrm{OT}=1.4(+/-2)$ and 19 control women age $=63$ $(+/-10), \mathrm{BMI}=26(+/-5), \mathrm{OT}=6.5(+/-7)$. The multivariate analysis adjusted for age, $\mathrm{BMI}$ and leptin levels, showed a significant lower level of OT in hand OA women $(p=0.002, \beta=3.4)$. In univariate analysis, there was no relationship between OT blood levels and the severity of OA (Verbruggen: $p=0.7, K L: p=0.2$ ), Joint Space Narrowing $(p=0.2)$ and osteophytes $(p=0.1)$.

Conclusions: Our study shows an anabolic effect of OT on chondrogenesis and that OT circulating levels was significantly lower in OA patients. OT might represents an interesting player in the pathophysiology of osteoarthritis and further studies will shed some light on its mechanism of action.

Disclosure of Interest: None declared

DOI: 10.1136/annrheumdis-2017-eular.6821 